\title{
Development of a 1.0 T MR microscope using a Nd-Fe-B permanent magnet
}

\author{
Tomoyuki Haishi, Takaaki Uematsu, Yoshimasa Matsuda, Katsumi Kose* \\ Institute of Applied Physics, University of Tsukuba, Tsukuba 305-8573, Japan
}

Received 2 September 2000; accepted 31 May 2001

\begin{abstract}
A compact 1.0 T MR microscope was developed using a permanent magnet made of high performance Nd-Fe-B magnetic material and a compact MRI console $(54 \mathrm{~cm}(\mathrm{~W}) \times 77 \mathrm{~cm}(\mathrm{H}) \times 60 \mathrm{~cm}(\mathrm{D}), 80 \mathrm{~kg}$ weight). Since the magnetic field of the permanent magnet had a large temperature coefficient $(-1200 \mathrm{ppm} / \mathrm{deg})$, an internal NMR locking technique was developed for the imaging sequences. The performance of the system was evaluated using several biological specimens. As a result, good SNR 3D images at $(50 \mu \mathrm{m})^{3}-(200 \mu \mathrm{m})^{3}$ voxel dimensions were obtained in practical imaging times (0.5-7.5 hours). Thus we have concluded that the permanent-magnet compact MR microscope has great promise as a research or analytical tool. (C) 2001 Elsevier Science Inc. All rights reserved.
\end{abstract}

Keywords: MR microscope; Permanent magnet; 3D image; Portable MRI console

\section{Introduction}

The development of high performance Nd-Fe-B magnetic material [1], of which stored magnetic energy is largest among practical materials for permanent magnets, has made it possible to construct whole body MRI magnets at a reasonable weight $(\sim 10$ tons) [2]. This permanent magnet technology has the potential to contribute to the development of new instruments for laboratories, such as an MR microscope.

For MR microscope systems, superconducting magnets have been almost exclusively used because of the potential high field strength (up to $17.6 \mathrm{~T}$ ) [3] and field stability. However, since there are many useful MR microscope applications in which spatial resolution of around 100 microns is sufficient, MR microscopes using permanent magnets have a great potential as research or analytical tools. However, because high-field permanent magnet circuits using Nd-Fe-B material have a large temperature coefficient (around $-1200 \mathrm{ppm} /$ degree), temperature drift of the Larmor frequency must be overcome to obtain MR microscope images at a high spatial resolution.

In the present study, we have developed a compact MR

\footnotetext{
* Corresponding author. Tel.: +81-298-53-5214; fax: +81-298-535205.

E-mail address: kose@ bk.tsukuba.ac.jp (K. Kose).
}

microscope using a $1.0 \mathrm{~T}$ permanent magnet and an internal NMR locking technique operated in a time-sharing mode. As a result we have successfully obtained 3D images at (50 $\mu \mathrm{m})^{3}-(200 \mu \mathrm{m})^{3}$ resolution within practical measurement times (0.5-7.5 hours).

\section{System hardware}

Figure 1 shows the overview of the MR microscope which we developed using a $1.0 \mathrm{~T}$ permanent magnet (Sumitomo Special Metals Corp., Osaka, Japan) and a compact MRI console. The specifications of the permanent magnet are shown in Table 1. Figure 2 shows the pole pieces of the magnet (outer diameters: $14 \mathrm{~cm}$, air gap: 61 $\mathrm{mm})$. The pole pieces consist of laminated silicon steel plates with radial cuts which can suppress the eddy currents caused by switching of the magnetic field gradients. As shown in the figure, the pole pieces each have a hollow region in the center. These hollow regions contribute to the improvement of the magnetic field homogeneity, in addition, many iron pieces are carefully placed in the hollow regions to improve the homogeneity of the magnetic field.

The compact MRI console is a portable system originally developed for an MR microscope which was used in conjunction with a magnetic field of an existing clinical MRI $[4,5]$. This console consists of four units: computer (PC), RF transceiver unit (modulator and detector), 3-channel gradi- 


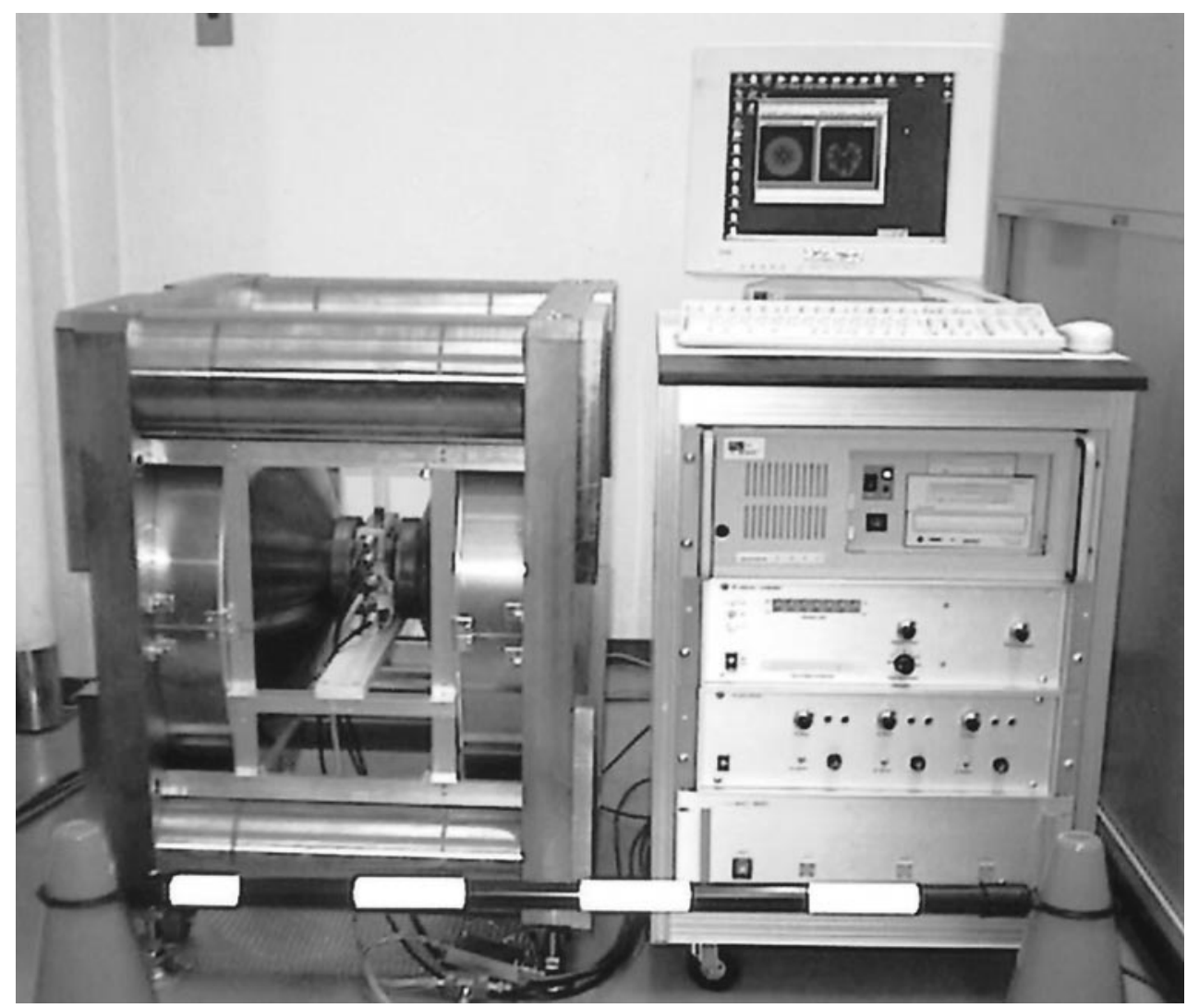

Fig. 1. MR microscope using a $1.0 \mathrm{~T}$ permanent magnet. This system can be installed within a $1.2 \mathrm{~m} \times 0.8 \mathrm{~m}$ area.

ent driver, and RF transmitter. Because the size of the console is $54 \mathrm{~cm}(\mathrm{~W}) \times 77 \mathrm{~cm}(\mathrm{H}) \times 60 \mathrm{~cm}(\mathrm{D})$ and the total weight is about $80 \mathrm{~kg}$, it can be moved easily.

All of the digital modules are contained inside the PC: DSP (digital signal processor) board (DSP6031, MTT Corp., Kobe, Japan) using a TMS320C31 DSP chip for the MRI pulse programmer [6] (100 ns time resolution), ADC board (PC-414G3, DATEL) for the data acquisition [7,8], and DDS (direct digital synthesizer) board for the RF signal source (FSW01, DST Inc., Japan). The PC system is running under Windows95 and is capable of real-time image reconstruction and display (about $50 \mathrm{~ms}$ for reconstruction and display after the data acquisition) $[7,8]$. The RF transceiver unit has an RF frequency range of 35-65 MHz.

Two gradient probes with different bore sizes were developed for this system. In both probes, the $G_{x}$ and $G_{y}$ coils are parallel four wire-coils $[9,10]$, the $G_{z}$ coils are Maxwell pairs, and the RF coils are solenoids. The small-bore probe is shown in Fig. 3. The gradient efficiencies of the probes are: $G_{x}=29, G_{y}=28$, and $G_{z}=26 \mathrm{mT} / \mathrm{m} / \mathrm{A}$ for the small-bore probe (I.D. $=6 \mathrm{~mm}$ ), and $\mathrm{G}_{\mathrm{x}}=12, \mathrm{G}_{\mathrm{y}}=11$, and $\mathrm{G}_{\mathrm{z}}=17 \mathrm{mT} / \mathrm{m} / \mathrm{A}$ for the large bore probe (I.D. $=20 \mathrm{~mm}$ ).

\section{NMR lock technique}

Because the magnetic material of the permanent magnet circuit has a high temperature coefficient (about -1200 $\mathrm{ppm} /$ degree), keeping the field strength-source frequency relationship constant is indispensable to MR microscopy. In the following subsections, the methods used to measure the magnetic field strength using a spin-echo signal, to correct the RF source frequency, and to keep the field-frequency relation constant during $3 \mathrm{D}$ image acquisition are presented.

\subsection{NMR lock using a spin-echo signal}

To measure the magnetic field strength precisely during imaging sequences, a spin echo signal of the imaged object itself was used. The Larmor frequency, relative to that of the RF signal source generated by the DDS synthesizer, was calculated using the time derivative of the quadraturephase (anti-symmetric) spin-echo signal at the spin-echo peak as shown in Fig. 4. The magnetic field strength or resonance frequency was calculated as the sum of the RF

Table 1

Specifications of the permanent magnet

\begin{tabular}{ll}
\hline Item & Specification \\
\hline Magnetic field strength & $0.968 \mathrm{~T}\left(22^{\circ} \mathrm{C}\right)$ \\
Air gap & $61 \mathrm{~mm}$ \\
Magnetic field homogeneity & $12 \mathrm{ppm}(20 \mathrm{~mm} \mathrm{dsv})$ \\
Size & $62 \mathrm{~cm}(\mathrm{~W}) \times 75 \mathrm{~cm}(\mathrm{H}) \times 75 \mathrm{~cm}(\mathrm{D})$ \\
Weight & $1,350 \mathrm{~kg}$ \\
\hline
\end{tabular}




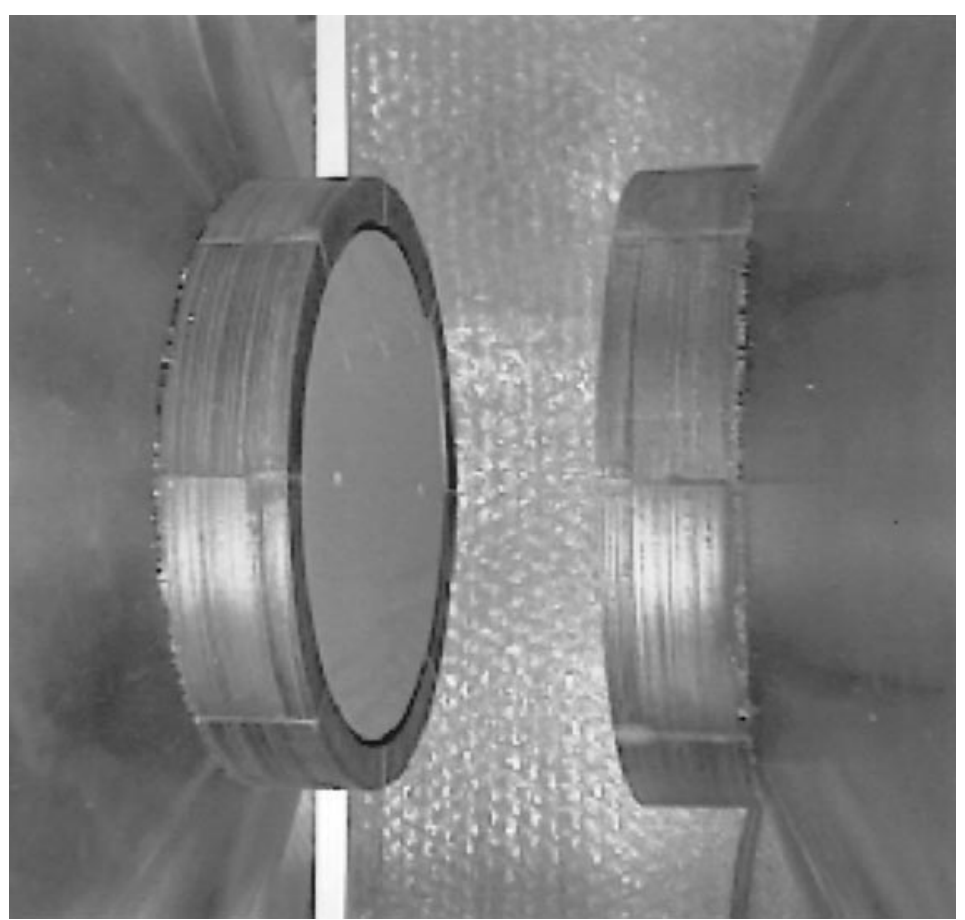

Fig. 2. Pole pieces of the magnet consist of 14-cm O.D. laminated silicon steel plates. The plates have radial cuts which suppress the eddy currents caused by gradient switching.

source frequency and the relative (or rotating frame) Larmor frequency. To establish the on-resonance condition, the new frequency value was updated to the DDS board interfaced via the ISA bus of the PC.
By using the above technique, the long term drift of the magnetic field was measured. Figure 5 shows the long term variation of the "locked" RF source frequency measured every three minutes with and without a thermal shield box

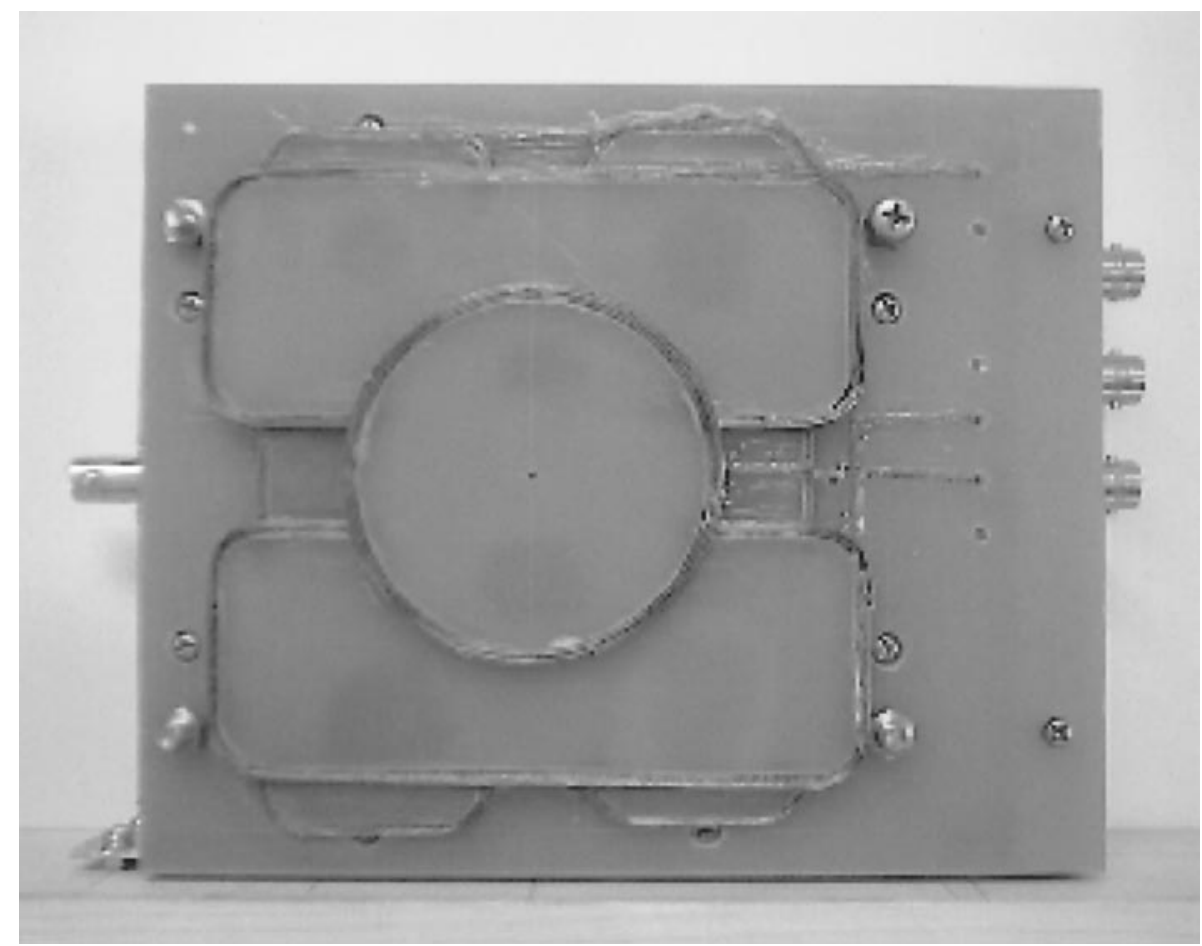

Fig. 3. Small bore gradient probe (dimension: $13 \mathrm{~cm}(\mathrm{H}) \times 13 \mathrm{~cm}(\mathrm{~W}) \times 4.5 \mathrm{~cm}(\mathrm{D})$ ). RF coil: solenoid (I.D. $=6 \mathrm{~mm}), \mathrm{G}_{\mathrm{x}}$ and $\mathrm{G}_{\mathrm{y}}$ : Anderson type, $\mathrm{G}_{\mathrm{z}}$ : Maxwell pair. The dimension of the large bore gradient probe is $16 \mathrm{~cm}(\mathrm{H}) \times 16 \mathrm{~cm}(\mathrm{~W}) \times 5.5 \mathrm{~cm}(\mathrm{D})$. 


\section{I phase signal}

\section{Q phase signal}

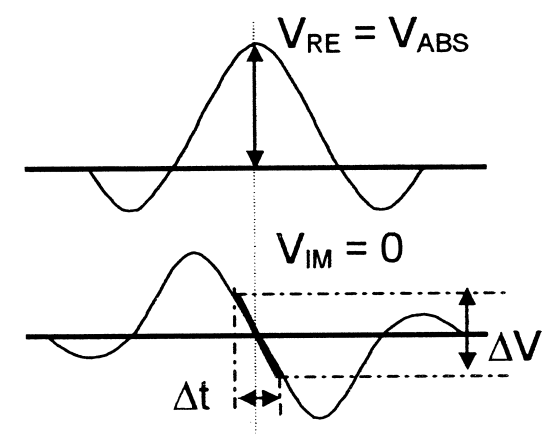

\section{Absolute value signal}

Fig. 4. The frequency deviation in the rotating frame was calculated using the time-derivative of the quadrature-phase signal at the spin-echo peak. The time for the spin echo peak was detected using the absolute value of the spin echo signal. The precision of the frequency depended on the SNR of the spin echo signal and was typically about $10 \mathrm{~Hz}(0.25 \mathrm{ppm}) . \mathrm{V}_{\mathrm{RE}}$ : voltage for in-phase (real-part) signal, $\mathrm{V}_{\mathrm{IM}}$ : voltage for quadrature-phase (imaginary-part) signal, $\mathrm{V}_{\mathrm{ABS}}$ : voltage for absolute value signal.

made of polystyrene foam slabs (30 mm thickness). When the magnet was covered with the thermal shield, the drift of the resonant frequency was around $500 \mathrm{~Hz} /$ hour, even if the room temperature was not regulated. Because our usual pixel bandwidth for 3D images was about $200 \mathrm{~Hz}$, the NMR reference frequency was locked at an interval of several minutes as described below.

\subsection{Combination of NMR lock and $3 D$ imaging sequence}

Figure 6 shows the time sequence for the NMR lock and the 3D imaging sequence: the NMR locking procedure was repeated just before each $2 \mathrm{D}$ plane acquisition in the 3D $\mathrm{k}$-space. This is because each 2D plane acquisition, usually takes several minutes, which is a suitable time interval for NMR locking as mentioned above.

\section{Results and discussion}

Imaging experiments were performed to evaluate the MR microscope system using several plant samples and chemically preserved mice. The parameters for the imaging sequences are shown in Table 2.

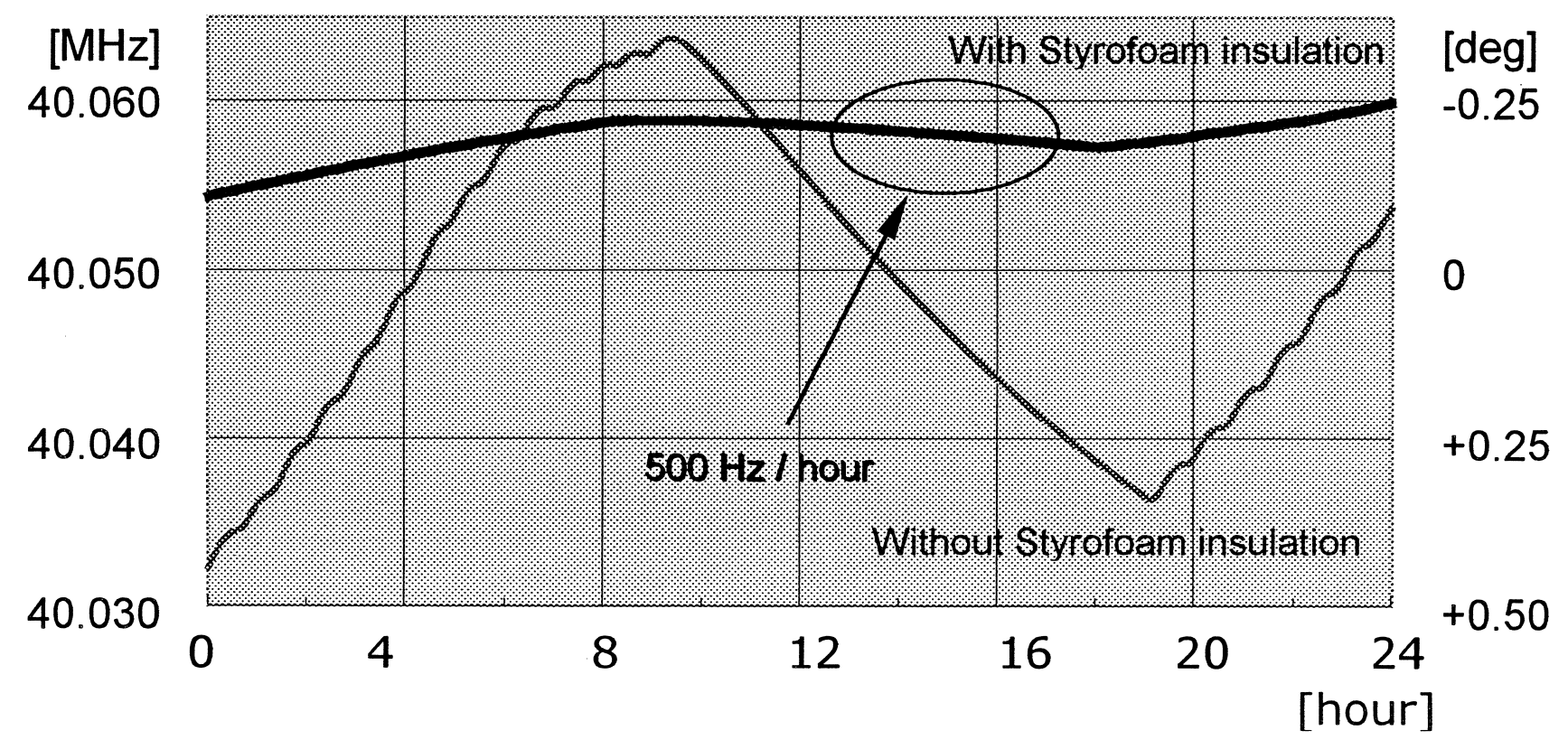

Fig. 5. Long term drift of the NMR frequency measured with the NMR lock technique. When the magnet was used with the styrofoam insulation, the frequency drift was about $500 \mathrm{~Hz} /$ hour. 


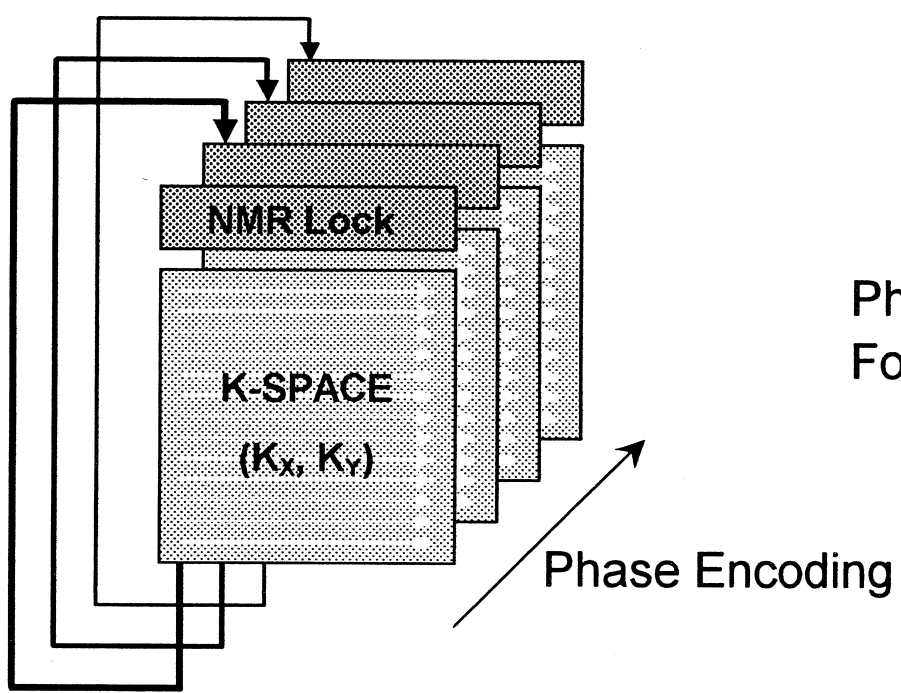

Phase Encoding For 3D Acquisitions

Fig. 6. 3D image acquisition sequence with the NMR lock sequence in the time-sharing mode. The field strength-source frequency relation is corrected before each k-space plane acquisition.

Figure 7 shows cross-sectional images of a garden pea and the root of a Welsh onion each acquired in about 7.5 hours. These images show that the 3D imaging sequence with the NMR lock sequence worked well and that several tens of microns spatial resolution is achievable for the proton-rich specimens. Figure 8 shows volume rendered images of a garden pea (a) and garden apple (b). These images show that this MR microscope gives adequate SNR for visualization of inner structures with approximately (100 $\mu \mathrm{m})^{3}$ voxel dimensions.

Figures 9 and 10 show cross-sectional images from 3D image data sets of the fixed mice. These images demonstrate that this MR microscope can be used for studies of chemically fixed animal samples.

As is well known, the intrinsic SNR of the NMR signal is proportional to the 7/4ths power of the magnetic field [11], if the RF coil is optimized. Thus the SNR of low-field MR microscopes (magnetic field $\sim 1 \mathrm{~T}$ ) is much lower than that of high-field MR microscopes $(\sim 10 \mathrm{~T})$ using superconducting magnets. However, the low-field MR microscopes have several advantages: $T_{1}$ is often shorter than that in high fields and $\mathrm{T}_{2}^{*}$ is much longer than that in high fields. From the latter reason, the bandwidth per pixel for the low field MR microscopes can be much narrower than that for the high-field MR microscopes. This can greatly contribute to improved SNR in low-field MR microscopes.

Furthermore, in our system, use of solenoid coils with many turns, which are only possible for the low resonance frequencies $(\sim 40 \mathrm{MHz})$ and small samples, has a great advantage. This is because small size solenoid coils have roughly a factor of three SNR advantage over saddle shaped coils [11] and many-turns solenoid coils can produce homogeneous RF fields, even if a coil is wound very close to the sample.

Besides the above advantages in MR measurements, the use of permanent magnet has another class of advantages: low cost comparing with superconducting magnets, no maintenance cost, and small space for installation.

In conclusion, we have developed a permanent-magnet MR microscope which is capable of observing biological samples with a resolution of approximately $(100 \mu \mathrm{m})^{3}$. The results suggest a great promise for this type MR microscope as a research or analytical tool.

Table 2

Parameters for the imaging condition

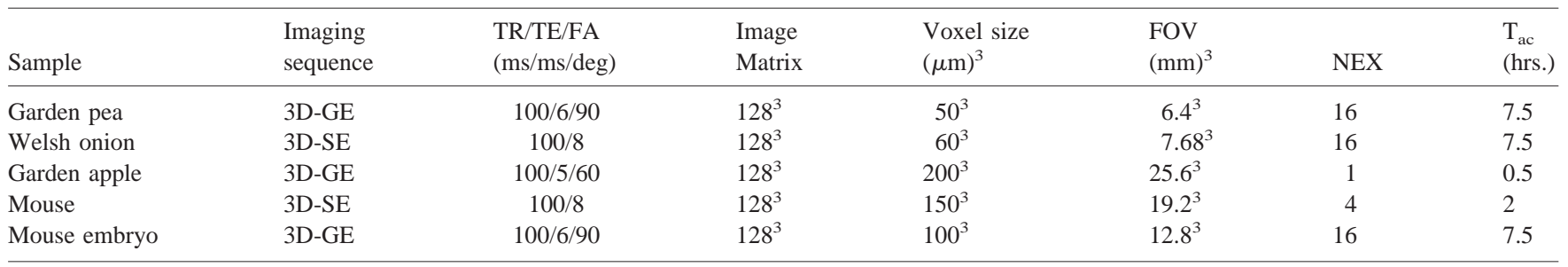

3D-GE: 3D gradient echo sequence, 3D-SE: 3D spin echo sequence, TR: repetition time, TE: echo time, FA: flip angle, NEX: number of excitation (signal accumulation), $\mathrm{T}_{\mathrm{ac}}$ : total measurement time. 


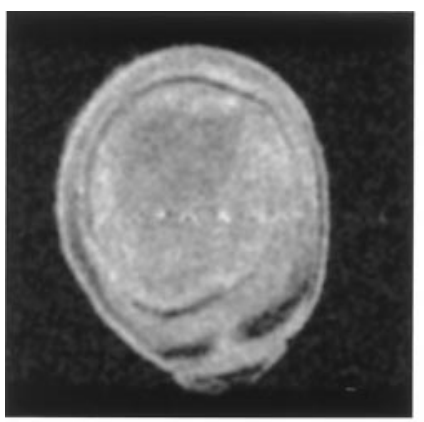

(a)

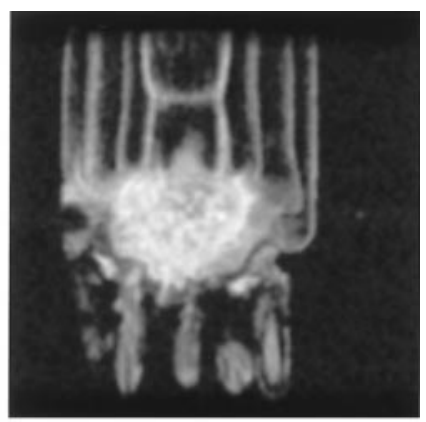

(b)

Fig. 7. (a) A cross sectional image of a garden pea acquired with a $3 \mathrm{D}$ gradient echo sequence $\left(\mathrm{TR} / \mathrm{TE} / \mathrm{FA}=100 \mathrm{~ms} / 6 \mathrm{~ms} / 90^{\circ}\right)$. Voxel size: $(50$ $\mu \mathrm{m})^{3}$. (b) A cross sectional image of a Walsh onion acquired with a $3 \mathrm{D}$ spin echo sequence $(\mathrm{TR} / \mathrm{TE}=100 \mathrm{~ms} / 8 \mathrm{~ms})$. Voxel size: $(60 \mu \mathrm{m})^{3}$.

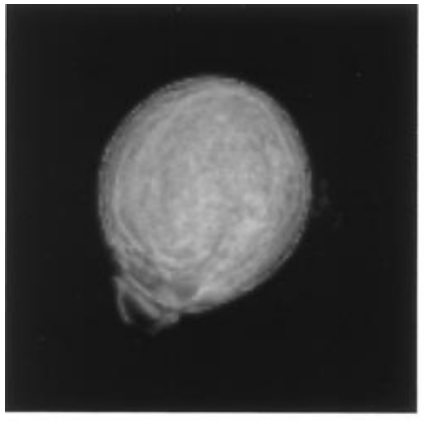

(a)

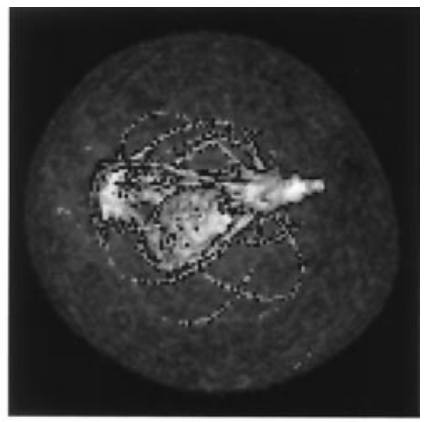

(b)

Fig. 8. (a) Volume rendering of a garden pea 3D image: $\mathrm{FOV}=(6.4 \mathrm{~mm})^{3}$ (b) Volume rendering of a garden apple 3D image: $\mathrm{FOV}=(25.6 \mathrm{~mm})^{3}$.

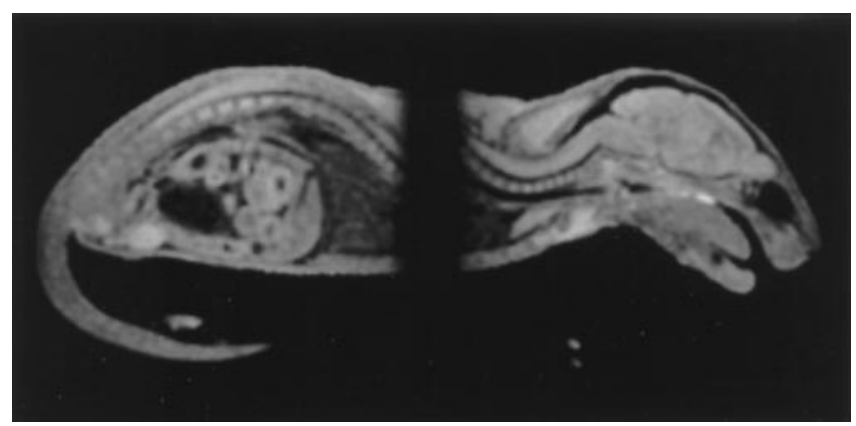

Fig. 9. Cross sectional images of a fixed mouse acquired with a 3D spin echo sequence $(\mathrm{TR} / \mathrm{TE}=100 \mathrm{~ms} / 8 \mathrm{~ms})$. Voxel size: $(150 \mu \mathrm{m})^{3}$. This is a composite image from two 3D data sets that were acquired separately.

\section{Acknowledgments}

We thank Mr. Y. Akita for construction of the large bore probe. We also thank Dr. Nakanishi and Mr. S. Okada at

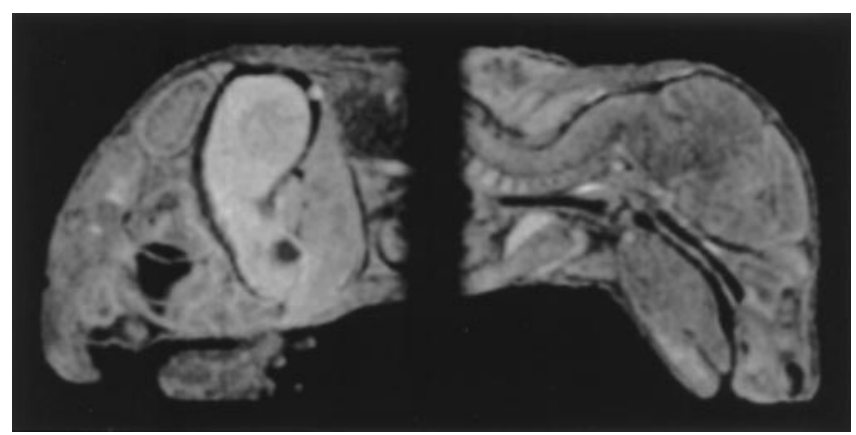

Fig. 10. Cross sectional images of a mouse embryo acquired with a 3D gradient echo sequence $\left(\mathrm{TR} / \mathrm{TE} / \mathrm{FA}=100 \mathrm{~ms} / 6 \mathrm{~ms} / 90^{\circ}\right)$. Voxel size: $(100$ $\mu \mathrm{m})^{3}$. This is a composite image from two 3D data sets that were acquired separately.

SSMC for stimulating discussion on the magnet. We finally thank Dr. R. Allen Waggoner at RIKEN for critical reading of the manuscript.

\section{References}

[1] Sagawa M, Fujimura S, Togawa N, Yamamoto H, Matsuura Y. New material for permanent magnets on a base of Nd and Fe. J Appl Phys 1984;55:2083-7.

[2] Miyamoto T, Sakurai H, Takabayashi H, Aoki M. A development of a permanent magnet assembly for MRI devices using Nd-Fe-B material. IEEE Trans. Magnetics 1989;25:3907-9.

[3] Roffmann WU, Gross D, Crozier S, Wilson S, Luescher K, Doddrell D, Lehmann V, Zick K. Hardware developments for in-vivo MR microscopy at fields up to 17.6 T. In Book of abstracts: Fifth International Conference on Magnetic Resonance Microscopy, L14, Heidelberg, 1999.

[4] Kose K, Haishi T, Adachi N, Anno I. A novel approach to the MR microscope: MR microscope with an independent console system (MRMICS) using a clinical whole body magnet. In: Book of abstracts: Sixth Annual Meeting of the Society of Magnetic Resonance in Medicine, Vol. 3. Berkeley, CA: ISMRM; 1998;1924.

[5] Yoshioka H, Kose K, Haishi T, Adachi N, Anno I, Itai Y. 2D and 3D microscopic imaging for biological tissues in vitro using a MR microscope with an independent console system (MRMICS). In: Book of abstracts: Seventh Annual Meeting of the Society of Magnetic Resonance in Medicine, Vol. 3. Berkeley, CA: ISMRM; 199;2119.

[6] Kose K, Haishi T. Development of a flexible pulse programmer for MRI using a commercial digital signal processor board. In: P. Blümler, B. Blümich, R. Botto, E. Fukushima (Eds). Spatially Resolved Magnetic Resonance, Weinheim: Wiley-VCH, 1998; pp. 703-9.

[7] Kose K, Haishi T, Caprihan A, Fukushima E. Real-time NMR imaging system using personal computers. J Magn Reson 1997;124:3541.

[8] Haishi T, Kose K. Real-time image reconstruction and display system for MRI using a high-speed personal computer. J Magn Reson 1998; 134:138-41.

[9] Anderson WA. Electrical current shims for correcting magnetic fields. Rev Sci Instrum 1961;32:241-50.

[10] Golay MJE. US Patent Application 733522, 1968.

[11] Hoult DI, Richards RE. The signal-to-noise ratio of the nuclear magnetic resonance experiment. J Magn Reson 1976;24:71-85. 